\title{
Probing the Molecular Mechanisms Governing the Oncolytic Activity of Paeonia suffruticosa on Triple-negative Breast Cancer Cells In Vitro
}

\author{
DONGYEON KIM ${ }^{1}$, DANIEL RADIN ${ }^{2}$ and DONNA LEONARDI ${ }^{3}$ \\ ${ }^{1}$ Department of Biology, College of Arts and Sciences, Washington University, St. Louis, MO, U.S.A.; \\ ${ }^{2}$ Department of Pharmacology, Stony Brook University School of Medicine, New York, NY, U.S.A.; \\ ${ }^{3}$ Bergen County Academies, Hackensack, NJ, U.S.A.
}

\begin{abstract}
Background/Aim: Extracts of Paeonia suffruticosa are traditionally used in Chinese medicine to increase blood flow. Recently, this extract has been shown to possess anti-tumor and anti-inflammatory properties, though this mechanism remains unknown. In the current work, we prepared extracts of $P$. suffruticosa and analyzed their effects on MDA-MB-231 triple-negative breast cancer cells. Materials and Methods: Varying concentrations of an aqueous extract of $P$. suffruticosa was administered to MDAMB-231. An MTS assay was used to determine the cell viability. Cytokine production was investigated through enzyme-linked immunosorbent assay (ELISA). Caspase-Glo assays were performed to measure caspase 3/7, 8 and 9 to analyze anti-apoptotic effects. Results: MTS assay for cell viability revealed that the extract increased viability at low concentrations $(0.6 \mathrm{mg} / \mathrm{ml})$ and decreased viability observed at concentrations $\geq 2.5 \mathrm{mg} / \mathrm{ml}(p<0.01)$. ELISA for $I L-6, I L$ 2 , and TNF-alpha revealed a biphasic dose-response inversely related to viability $(p<0.05)$. IL-24 expression also increased at $2.5 \mathrm{mg} / \mathrm{ml}$ and $4.0 \mathrm{mg} / \mathrm{ml}(p<0.05)$. Bax levels remained relatively constant while Bcl-2 decreased significantly in all concentrations $(p<0.01)$. Small decreases in Fas ligand levels was observed in parallel with a lack of increase in caspase-8 activity. Most notable was that while $4 \mathrm{mg} / \mathrm{ml}$ of $P$. suffruticosa extract reduced MDA-MB-231 viability by $>60 \%(p<0.01)$, the same concentration reduced the viability of non-transformed HaCat cells by $\sim 8 \%$ $(p>0.05)$, suggesting a selective oncolytic effect. Conclusion: $P$. suffruticosa extract has the ability to modulate the
\end{abstract}

Correspondence to: Daniel Pierce Radin, Department of Biology, College of Arts and Sciences, University of Rochester, Rochester, 14627, NY, U.S.A. Tel: +1 2016790671, e-mail: danradin1@gmail.com

Key Words: Apoptosis, cytokine, drug discovery, Paeonia suffruticosa, triple-negative breast cancer. production of several tumor suppressive cytokines, induce intrinsic apoptosis and has the capability of reducing cancer burden while sparing healthy tissue.

Breast cancer is the most common type of cancer affecting women in the United States and is the leading cause of cancer death among women of all races. About 1 in 8 U.S women will develop breast cancer over the course of their lifetime. In 2010, breast cancer was diagnosed in over 1.5 million women, and over 500,000 women worldwide died of this disease (1). Treatment of the disease most often incorporates the use of chemotherapy, targeted therapy, or hormonal therapy. Approximately $15-20 \%$ of breast cancers are categorized as having a triple-negative phenotype because they lack estrogen, progesterone, and HER2/neu receptors. These cancers generally have a poor prognosis because there is no clinically-validated hormonal or targeted therapy. The available cytotoxic and genotoxic drugs produce limited efficacy and significant side effects (2). Since the adverse effects of the various drug regimens place limitations on their therapeutic use, there is an urgent need in developing effective agents for triple negative breast cancer, which reduce these adverse effects (3). Novel agents with multiple targeting abilities distinct from the known targets could be useful for circumventing the limitations of current treatment options.

There is increasing evidence that in U.S.A., cancer patients take botanical supplements concurrently with their chemotherapeutic agents (4). Studies on botanicals show that they could possess effective anticancer compounds that may be used alone or as adjuvants to existing chemotherapy regimens to improve efficacy and/or reduce drug-induced adverse effects (5). The use of herbals as adjuvants has been shown to produce beneficial effects in human malignancies such as liver, esophageal, prostate and lung cancer, as well as reduce the symptoms associated with various cancers $(3,6,7)$. However, the cellular 
mechanisms underlying the effects of these natural products are most often unclear and the identification of their biologically active compounds is of great significance to pharmaceutical research (8).

Paeonia suffruticosa is traditionally used as a Chinese herbal medicine to increase the blood flow and decrease blood stasis from stagnation. While scant in literature, previous studies have shown that extracts of $P$. suffruticosa exhibit potential in the therapy and prevention of several major human diseases including cancers of several etiologies (6). Extracts of P. suffruticosa are also used as herbal medicines to treat various diseases such as macula, epilepsy, menstrual disorders, atherosclerosis, infection, inflammation, and cutaneous diseases $(8,9)$. Studies report that extract of $P$. suffruticosa has potent anti-oxidant, anti-mutagenic, antiproliferative, anti-invasive, anti-infective, anti-hypertensive, anti-arrhythmic, anti-inflammation, anti-diabetic, anti-obesity and neuroprotective activities, but of unknown etiology (810). In the present study, the anti-proliferative effects and the mechanism of action of an aqueous extract of $P$. suffruticosa were investigated in vitro on a triple negative breast cancer cell line, MDA-MB-231.

\section{Materials and Methods}

Preparation of aqueous extract of P. suffruticosa. Aqueous extracts of P. suffruticosa (Omniherb, Daegu, Republic of Korea) were prepared by boiling $200 \mathrm{~g}$ of dried root in $500 \mathrm{~mL}$ of distilled water for $1 \mathrm{~h}$ at $100^{\circ} \mathrm{C}$. The aqueous extract was filtered through a $.45 \mu \mathrm{m}$ filter and was lyophilized overnight. $800 \mathrm{mg}$ of $P$. suffruticosa was reconstituted in $1 \mathrm{~mL}$ of water and concentrations ranging from $800 \mathrm{mg} / \mathrm{mL}$ to $30 \mathrm{mg} / \mathrm{mL}$ were created. When administered in culture, concentrations were reduced 100 -fold due to dilution in the cell culture media.

Cell culture and treatment. MDA-MB-231 (MDA) (estrogen, progesterone, and HER2/neu receptor negative) and $\mathrm{HaCaT}$ (human keratinocyte) cell lines were purchased from the American Type Culture Collection (Manassas, VA, USA). The cells were grown in Dulbecco's Modified Eagle's Medium (DMEM)/F12, supplemented with $10 \%$ heat-inactivated fetal bovine serum, and $1 \%$ penicillin/streptomycin in an incubator at $37^{\circ} \mathrm{C}$ with atmospheric $\mathrm{CO}_{2}$ of $5 \%$ and $100 \%$ humidity. $24 \mathrm{~h}$ after plating $(200,000$ cells $/ \mathrm{mL} ; 20,000$ cells/well in 96 well plate) cells were treated with the extract for $48 \mathrm{~h}$, after which cells were assayed or lysed depending on the individual experiment.

MTS assay for cell viability. The effects of $P$. suffruticosa treatment on cellular proliferation of MDA-MB-231 and human keratinocyte $\mathrm{HaCaT}$ cells was measured using an MTS (3-(4,5-dimethylthiazol2-yl)-5-(3-carboxymethox y phenyl)-2-(4-sulfophenyl)-2Htetrazolium) assay. Cells were seeded at a density of 20,000 cells per well in $100 \mu \mathrm{L}$ of medium. Fifteen microliters of CellTiter $96^{\circledR}$ Aqueous One Solution Cell Proliferation Assay kit (Promega, Madison, WI, USA) was added to each well. The cells were incubated for $1 \mathrm{~h}$ at $37^{\circ} \mathrm{C}$, during which time the tetrazolium compound in the reagent was reduced to formazan, producing a colorimetric response proportional to the number of viable cells in each well. The absorbance was measured at 490nm with an ELx808 microplate reader (BioTek, Winnoski, VT, USA).

Preparation of cell lysis. MDA-MB-231 cultured in T-25 flasks (Corning, Corning, NY, USA) were treated with P. suffruticosa at concentrations of $0.6,2.5,4.0 \mathrm{mg} / \mathrm{mL}$. After $48 \mathrm{~h}$, the flasks were trypsinized and centrifuged at $1200 \mathrm{rpm}$ for $7 \mathrm{~min}$ at $25^{\circ} \mathrm{C}$. Cells were washed in $1.5 \mathrm{~mL}$ of ice-cold $1 \mathrm{X}$ PBS (pH 7.4; Invitrogen) and centrifuged again at $1200 \mathrm{rpm}$ for $7 \mathrm{~min}$. After supernatants were discarded, ice-cold 1X lysis buffer (Cell Signaling Technology, Danvers, MA, USA) with $10 \mu \mathrm{L}$ of protease inhibitor cocktail (Sigma-Aldrich, St. Louis, MO, USA) per $1 \mathrm{~mL}$ lysis buffer was added to cells lysed at $1 \times 10^{7}$ cells $/ \mathrm{mL}$. The cells were chilled on ice for $10 \mathrm{~min}$ and then centrifuged at $12000 \mathrm{rpm}$ for $15 \mathrm{~min}$ at $4^{\circ} \mathrm{C}$. The cell lysates were collected in $1.5 \mathrm{~mL}$ microcentrifuge tubes and stored at $-80^{\circ} \mathrm{C}$.

Enzyme-linked Immunosorbent Assay (ELISA). To measure production of IL-6, IL-2, TNF-alpha, IL-24, Bax and Bcl-2 production in MDA-MB-231 cells, an indirect ELISA was performed using the Protein Detector HRP ELISA (KPL, Gaithersburg, MD, USA) as per the manufacturer's instructions. Primary polyclonal rabbit antibodies for IL-6 (Abcam, Cambridge, MA, USA), IL-2 (Abcam, Cambridge, UK), TNF-alpha (Abcam), IL-24 (Santa Cruz Biotechnology, Santa Cruz, CA, USA), Bax (Santa Cruz Biotechnology, Santa Cruz, CA, USA), Bcl-2 (Santa Cruz Biotechnology, Santa Cruz, CA, USA), and cyclin D1 (Santa Cruz Biotechnology, Santa Cruz, CA, USA) as well as the secondary HRP-labeled antibody (KPL) were each diluted at 1:500 in $1 \mathrm{X}$ BSA Diluent/Blocking Solution. When measuring secreted cytokines, percent of control was calculated per remaining cell as determined by viability assay. Absorbance of each well was measured at $405 \mathrm{~nm}$ in a microplate-reader.

Caspase 3/7, 8, and 9 assay. The Caspase-Glo Assays (Promega, Madison, WI, USA) were performed to measure caspase $3 / 7,8$ and 9 to analyze anti-apoptotic effects of the treatment. Caspase-Glo Reagent was prepared as per company assay instructions. Reagent $(100 \mu \mathrm{L})$ was added to each well of a black 96-well plate, which had been seeded at 20,000 cell/well and treated for $48 \mathrm{~h}$ as previously described. The plate was incubated for $45 \mathrm{~min}$ at $37^{\circ} \mathrm{C}$ and $5 \% \mathrm{CO}_{2}$ and luminescence was measured using a microplate reader. Values were normalized to luminescence of cells treated with the vehicle.

Statistical analysis. T-tests were used to determine statistical significance. Analysis of variance was done using staplus to determine overall trends. Alpha value was set at 0.05 . $(n=4-8)$ refers to data points per concentration and all assays were performed 2 or more times.

\section{Results}

P. suffruticosa is selectively oncolytic. Varying concentrations of an aqueous extract of $P$. suffruticosa was administered to MDA-MB-231 cells. An MTS assay was used to determine the cell viability after $48 \mathrm{~h}$ of treatment. Exposure to $P$. suffruticosa produced a biphasic dose-response with cell proliferation at low concentrations $(0.6 \mathrm{mg} / \mathrm{ml})$ and reduced cell viability at concentrations greater than $2 \mathrm{mg} / \mathrm{ml}$ (Figure 
A

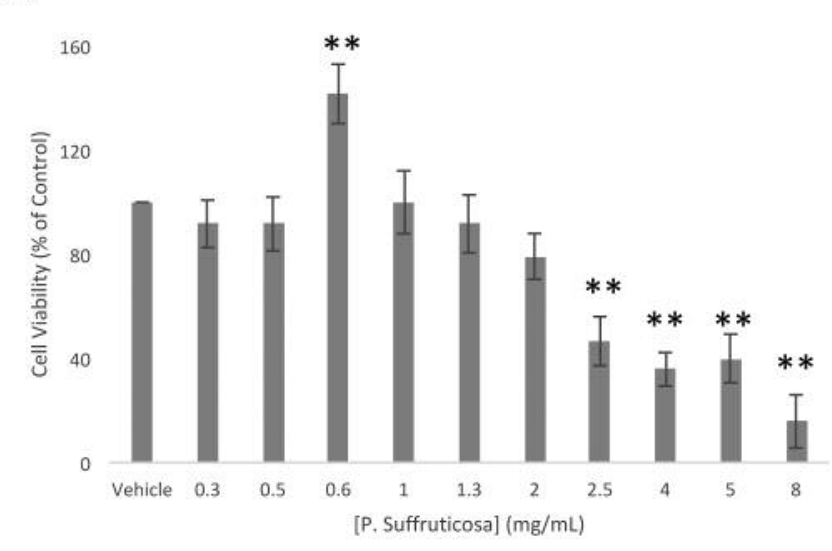

C

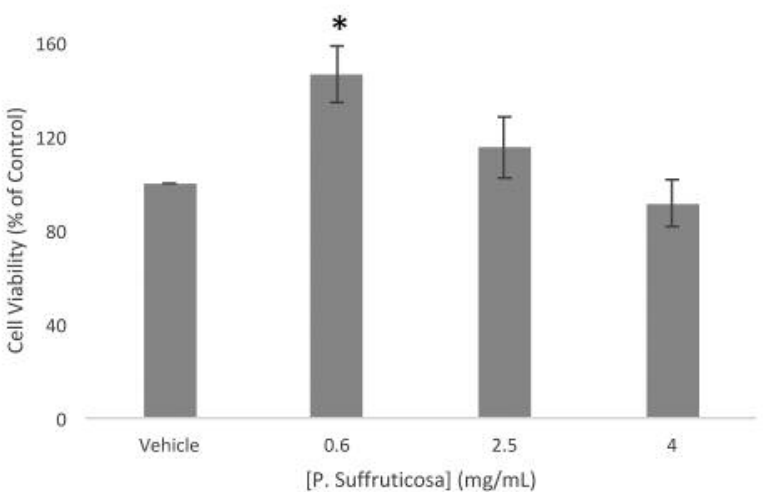

B
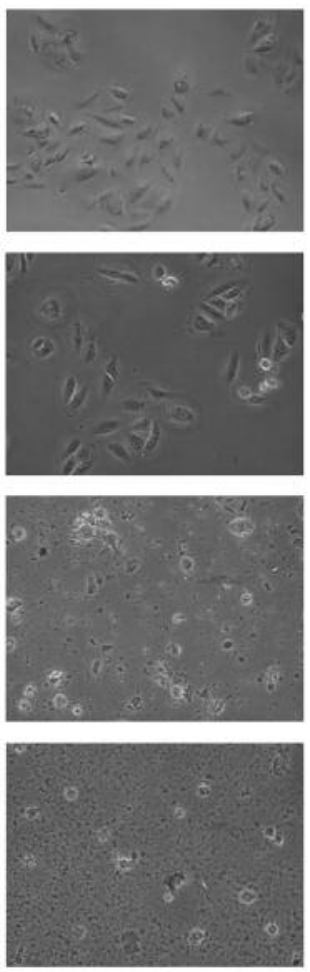

Figure 1. P. suffruticosa extract is selectively cytotoxic. A) Effects of P. suffruticosa extract on MDA-MB-231 cell viability after 48 hours. B) Light microscope pictures of MDA-MB-231 cells treated with vehicle, $0.6,2.5$, and $4 \mathrm{mg} / \mathrm{ml}$ of extract. C) P. suffruticosa extract is not lethal to nontransformed HaCat cells. Results from 2-3 separate experiments are shown as mean $\pm S D(n=5)$. ${ }^{*}<<0.05$, **p<0.01, t-test comparing data to that of vehicle control.

1a). Light microscopy pictures revealed significant rounding and granularity at $2.5 \mathrm{mg} / \mathrm{ml}$ and $4 \mathrm{mg} / \mathrm{ml}$ (Figure $1 \mathrm{~b}$ ), in addition to a general loss of healthy cells, in line with data suggesting that these concentrations significantly reduce cell viability of MDA-MB-231. Most notably, treatment of nontransformed HaCat cells with $P$. suffruticosa revealed a biphasic dose-dependent effect. At $0.6 \mathrm{mg} / \mathrm{ml}, P$. suffruticosa elicited a pro-proliferative response (Figure 1c, $p<0.05$ ). Most notably, 2.5 and $4 \mathrm{mg} / \mathrm{ml} P$. suffruticosa, concentrations that significantly reduce MDA-MB-231 cell viability, do not significantly reduce $\mathrm{HaCat}$ cell viability, indicative of a selective oncolytic effect.

The Effect of P. suffruticosa on cytokine production in MDA$M B-231$. Cytokine production by MDA-MB-231 after treatment with $P$. suffruticosa for $48 \mathrm{~h}$ was investigated through enzyme-linked immunosorbent assay (ELISA) (Figure 2a). Interleukin 6 and interleukin 2 are naturally occurring proteins that mediate communications between cells, regulate cell growth, differentiation and motility and stimulate immune responses $(11,12)$. Tumor necrosis factor alpha (TNF-alpha) plays a key role in apoptosis and is currently used for treatment in cancer of locally advanced soft tissue sarcomas and metastatic melanomas (13). IL-6, IL-2 and TNF-alpha at $0.6 \mathrm{mg} / \mathrm{ml}$ decreased significantly, but subsequently increased at concentrations greater than $2.5 \mathrm{mg} / \mathrm{mL}(p<0.05)$ (Figure $2 a)$. Interestingly, these cytokine levels were inversely proportional to the viability graph exhibiting the bi-phasic dose response shown in Figure 1.

Studies have shown that interleukin-24 is up-regulated through increased IL-2 production (14). The levels of IL-24 after treatment for $48 \mathrm{~h}$ were identified to be significantly different from the control at 2.5 and $4.0 \mathrm{mg} / \mathrm{ml}$ when measured by an indirect ELISA $(p<0.01)$ (Figure 2b). This 
A

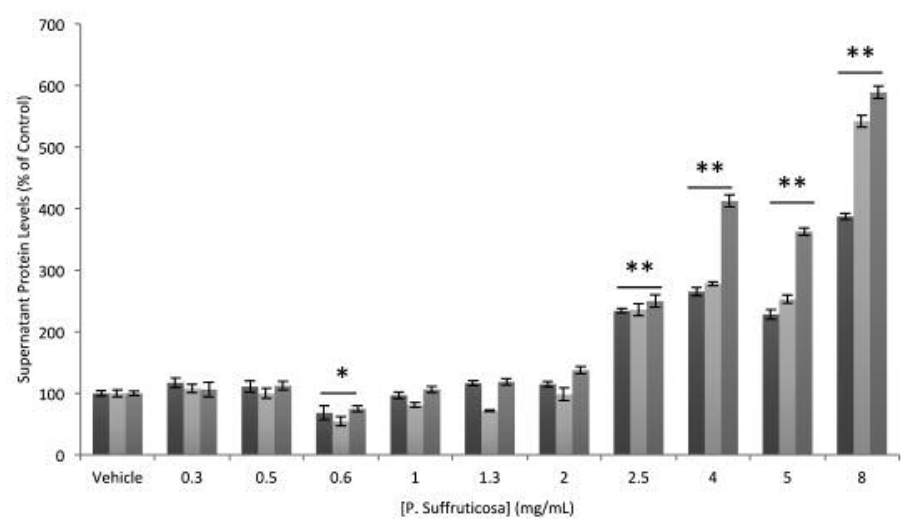

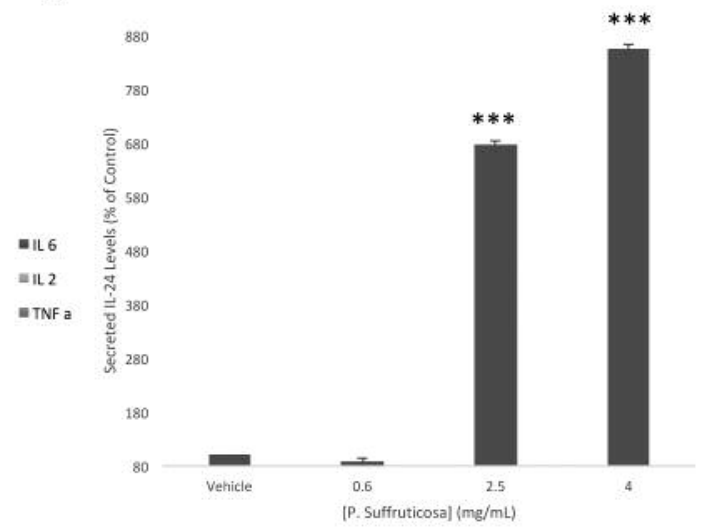

Figure 2. P. suffruticosa extract modulates the levels of several cytokines in MDA-MB-231 in a dose-dependent fashion. A) Levels of IL-6, IL-2 and $T N F$ - a were measured after 48 h of $P$. suffruticosa extract treatment and normalized to levels observed after treatment with vehicle control. ${ }^{*} p<0.05$ ${ }^{* *} p<0.01, t$-test showing levels of all cytokines at that dose to be significantly different than levels of vehicle control. B) $P$. suffruticosa extract modulates levels of the tumor suppressor IL-24 in a biphasic manner. $* * * p<0.001, t$-test comparing data to that of vehicle control. Results from two or three separate experiments are shown as mean $\pm S D$.
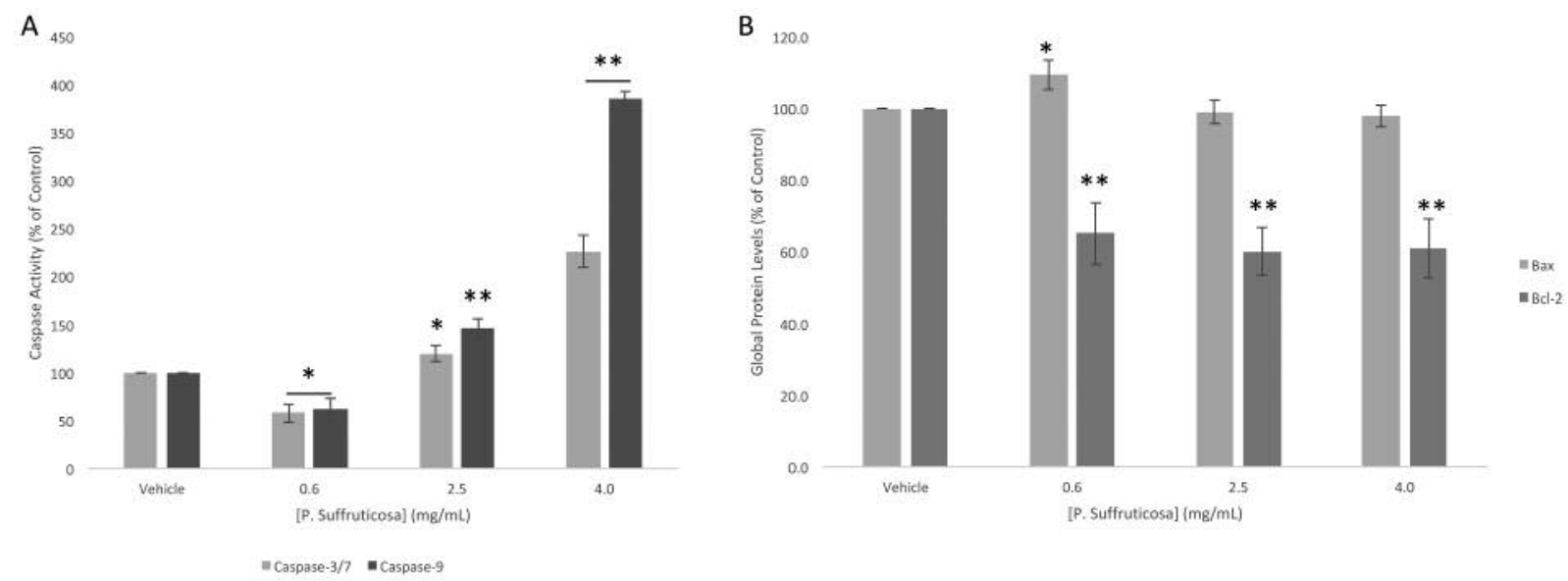

Figure 3. P. suffruticosa extract modulates factors indicative of apoptosis in MDA-MB-231 cells. A) P. suffruticosa extract modulates active caspase9 and $-3 / 7$ levels in a biphasic manner. ${ }^{*} p<0.05$, ${ }^{*} p<0.01, t$-test showing levels of active caspases at that dose to be significantly different than levels of vehicle control. ${ }^{*} p<0.05$, ** $p<0.01$, t-test comparing data to that of vehicle control. B) P. suffruticosa extract modulates levels of BAX and BCL-2, cellular governors of intrinsic apoptosis. ${ }^{*} p<0.05$, ${ }^{*} p<0.01$, t-test comparing data to that of vehicle control. Results from $2-3$ separate experiments are shown as mean $\pm S D$.

increase in IL-24 suggests $P$. suffruticosa may be stimulating its anti-proliferative effects through IL-24. IL-24 has been reported to induce apoptosis, both extrinsically through the up-regulation of Fas ligand (15), as well as intrinsically through decreasing pAKT (16).

High-dose P. suffruticosa treatment induces apoptosis through caspase 3/7 and 9 in MDA-MB-231. In order to further investigate the extrinsic $v s$. intrinsic mechanism of apoptosis by the aqueous extract, caspase $3 / 7,8$ and 9 were investigated following treatment with P. suffruticosa (Figure 3a). Caspase $3 / 7$ is the ultimate death enzyme and is involved in the processing of upstream caspase 9, which mediates apoptosis predominantly intrinsically, and caspase 8, which mediates apoptosis through predominantly extracellular mechanism (17). There was an increase in caspase $3 / 7$ and 9 activity at 2.5 and $4.0 \mathrm{mg} / \mathrm{ml}$ after treatment in a dose dependent fashion. Caspase $3 / 7$ and 9 all 
decreased at concentration of $0.6 \mathrm{mg} / \mathrm{ml}$, following the biphasic curve of proliferation seen in Figure 1. Caspase-8 activity was observed to decrease or remain at vehiclecontrol levels at every concentration (data not shown). The increase in caspase 9 coupled with decrease in caspase 8 indicates a mechanism of action of apoptosis that is intrinsic possibly mediated through IL-24 (Figure 2b).

To gain more perspective on the molecular mechanisms of Caspase activation after $P$. suffruticosa treatment, we assayed for changes in the production of proteins canonically related to intrinsic and extrinsic cell death. In line with a lack of Caspase- 8 activity, membrane bound and secreted Fas ligand levels did not decrease or were observed to decrease slightly (data not shown), again confirming the lack of death receptor participation in the mechanism by which $P$. suffruticosa selectively eliminates MDA-MB-231 cells.

We also assayed for changes in the ratio of BAX to BCL2 , as modulation of the ratio of these two proteins is a main determinant in propensity for intrinsic apoptosis. As can be seen in Figure 3b, Bax production levels increased at 0.6 $\mathrm{mg} / \mathrm{ml}$ but were not significantly different at 2.5 and 4.0 $\mathrm{mg} / \mathrm{ml}$ of $P$. suffruticosa. However, Bcl-2 levels decreased at all concentrations $(0.6,2.5,4.0 \mathrm{mg} / \mathrm{mL})(p<0.01)$. The anti-apoptotic protein (Bcl-2) levels decreased significantly while pro-apoptotic protein (Bax) levels remained similar to the control at concentrations of 2.5 and $4.0 \mathrm{mg} / \mathrm{ml}$, indicating occurrence of mitochondrial-dependent apoptosis. It is also curious that the Bax/BCL- 2 raito was altered significantly at $0.6 \mathrm{mg} / \mathrm{ml}$, yet cell viability was not diminished at this concentration (Figure 1a).

\section{Discussion}

Medicinal plants are considered to be a main source of biologically-active compounds that can be used for the treatment of various ailments including cancer. Studies have demonstrated anticancer activities of various herbal remedies in leukemia, colorectal carcinoma, gastric cancer, and prostate cancer (6). In previous studies, Paeonia suffruticosa, a naturally-occurring plant lignin, was shown to have antiproliferative effects in liver, esophageal and lung cancer. However, its cellular mechanisms of action have never been thoroughly described. The present study was designed to understand the mechanisms by which $P$. suffruticosa might act as an anticancer agent and to draw attention to its natural anti-proliferative properties. The model used was a triple negative breast cancer cell line, MDA-MB-231, which is a very aggressive form of breast cancer with limited therapeutic options.

Inhibition of cell proliferation and induction of apoptosis are characteristics that any chemotherapeutic agent must possess. The data reported here (Figure 1) demonstrated that an aqueous extract of $P$. suffruticosa significantly inhibited cell viability in MDA-MB-231 cells in a biphasic dose response manner, which suggests $P$. suffruticosa may have apoptotic effects against triple negative breast cancer at higher concentrations $(2.5 \mathrm{mg} / \mathrm{ml}-8.0 \mathrm{mg} / \mathrm{ml})$. However, at a low dose, $P$. suffruticosa induced cell proliferation. This low dose proliferative response, although not uncommon for many molecules, warrants further investigation. What is of particular clinical interest was that concentrations that reduced cell viability of MDA-MB-231 cells (2.5 and $4 \mathrm{mg} / \mathrm{ml}$ of extract) did not significantly reduce viability of non-transformed $\mathrm{HaCat}$ cells, indicating that the active compounds from this extract selectively eliminate cancer cells, which may make this extract an attractive candidate for clinical development for the treatment of neoplastic disease.

Promoting apoptotic protein production in cancer cells is a key goal in cancer therapy. Therefore, inhibiting anti-apoptotic proteins may allow cells to undergo apoptosis. To determine if $P$. suffruticosa induces apoptosis rather than necrotic cell death in MDA-MB-231 cells, the levels of Bax and Bcl-2 production were measured after treatment of $P$. suffruticosa $(0.6 \mathrm{mg} / \mathrm{ml}-4 \mathrm{mg} / \mathrm{ml})$. The ratio of these two proteins is often considered to control the apoptotic status of the cell. After 48 $\mathrm{h}, \mathrm{Bcl}-2$, an anti-apoptotic protein, decreased across all concentrations of $P$. suffruticosa while Bax, a pro-apoptotic protein, remained relatively constant (Figure $3 b$ ). The decrease in Bcl2:Bax ratio indicated onset of apoptosis by $P$. suffruticosa. It should be noted that at the lowest concentration of $P$. suffruticosa, or at its proliferative dose, Bax increased significantly, but proliferation actually ensued.

The cytokine data obtained in this research also supported the anti-proliferative effects of the aqueous extract of $P$. suffruticosa, inversely following the biphasic viability data (Figure 1). TNF-alpha, which has been proven to play a key role in inducing apoptosis in cancer (13) increased significantly at higher concentrations (Figure 3). Although TNF-alpha is also known for its role in inflammation and immunity in normal cells (18), P. suffruticosa may initiate cell death through up-regulation of TNF-alpha. Increase in IL-6, an inflammatory cytokine, could represent an avenue to apoptosis through oxidative stress. Existing chemotherapeutic agents, such as cisplatin, rely on generation of reactive oxygen species for cytotoxicity (19). However, this increase in IL-6 should be further investigated since chronic inflammation is often considered significant to cancer progression due to the promotion of these reactive oxygen species especially in compromised cells. IL-2 levels also increased significantly at higher concentrations of extract (Figure 2), which may be an important mechanism in $P$. suffruticosa's anti-proliferative effect. IL-2 has been FDA approved as an immunotherapy for the treatment of malignant melanoma and renal cell cancer in large intermittent toxic doses (20). The increased levels of IL2 may be a mechanism of action of $P$. suffruticosa although MDA-MB-231 is only an in vitro model without immune cell 
stature. This may be due to the fact that MDA-MB-231 is constitutively producing IL-2 and perhaps signaling for the modulation of related proteins in an autocrine fashion.

Previous research has suggested that increased levels of IL-2 can result in the up-regulation of IL-24 (14). A study by Jen et al. demonstrated that one of the mechanisms of effective IL-2 therapy is through direct up-regulation of the tumor suppressor IL-24 in melanoma tumor cells, which resulted in growth suppression. The protein encoded by this gene has been shown to induce apoptosis selectively in various cancer cells in several ways but literature is scant. The intriguing increase in IL-2, with a corresponding significant decrease in cell viability at concentrations of $P$. suffruticosa greater than $2.5 \mathrm{mg} / \mathrm{ml}$ supports this previous research (Figure 3). After cells were treated with $P$. suffruticosa for $48 \mathrm{~h}$, it was found that IL-24 levels were significantly increased above control in the higher concentrations that resulted in reduced viability (Figure 5). This suggests that one of the mechanisms of action of $P$. suffruticosa's anti-proliferative effects could be through the increased levels of IL-24, a tumor suppressor. This should be considered a very significant finding.

In the study reported here, Fas ligand secretion was then observed to determine a potential mechanism of $P$. suffruticosa through IL-24. IL-24 was previously shown to inhibit cell proliferation by activation of the Fas-FasL signaling pathway (15). However, after the exposure to $P$. suffruticosa, the production of Fas Ligand decreased slightly (data not shown). Fas Ligand induces apoptosis extrinsically, suggesting that the induction of cell death by $P$. suffruticosa was not extrinsically activated by Fas/Fas ligand apoptotic death. It is also important to note that MDA-MB-231 cells are particularly resistant to Fas-mediated apoptosis due to up-regulation of FAIM2 (21). Previous research also demonstrated that synergistic antitumor activity elicited by IL-24 was associated with the activation of extrinsic as well as intrinsic apoptotic pathways (22). This warranted further study into caspase levels, specifically caspase 9 and caspase 8 . Notably, both pathways converge at caspase $3 / 7$, leading to activation of other proteases. The extrinsic death receptor pathway is triggered by Fas ligand and proceeds through caspase- 8 to activate executor caspases through caspase $3 / 7$. The intrinsic pathway is triggered through mitochondrial outer membrane permeabilization, resulting in the release of cytochrome $c$ from mitochondria, apoptosome assembly, and activation of caspase 9 and caspase 3/7 (23). The aqueous extract of $P$. suffruticosa caused a statistically significant increase in caspase 9 and 3/7 at the concentrations of extract that caused apoptosis (Figure 3a). In addition, caspase 8, 9 and 3/7 decreased at lower concentrations (Figure 3a, data not shown) where cell proliferation was noted, supporting the previous result of bi-phasic dose response of $P$. suffruticosa on cell viability (Figure 1). Caspase 8 decreased in all concentrations except at the highest concentration tested, which corroborates with the results of Fas Ligand production (data not shown). Therefore, it was concluded that $P$. suffruticosa does not induce apoptosis through an extrinsic pathway in MDA-MB-231, but through an intrinsic pathway, activating caspase 9 (Figure 3).

Although previous studies showed that P. suffruticosa inhibits proliferation in various cancer cell lines, its mechanism of action still remained unclear. This study highlights, for the first time, the potential of $P$. suffruticosa as a triple negative breast cancer therapy. While in vitro, it was concluded that the anti-proliferative effect of $P$. suffruticosa at high concentrations is most likely due to the up-regulation of IL-2. The increase in IL-2 levels was corroborated with up-regulation of IL-24, a tumor suppressor. It was concluded that the up-regulation of IL-24 and the reduction of BCL-2 could lead to apoptosis, suggesting one of $P$. suffruticosa's principal mechanisms of action. $P$. suffruticosa also was concluded to induce apoptosis intrinsically through the increased levels of caspase 3/7 and 9. Exposure to $P$. suffruticosa had no effect on cell viability in keratinocytes, signifying its selective effect on cancer cells. It was concluded that $P$. suffruticosa extract has potential as an anti-tumor agent by exhibiting anti-proliferative effects as well as apoptotic effects. Future studies are warranted in determining the interaction of $P$. suffruticosa with known chemotherapeutic drugs for triple-negative breast cancer cells. This study also provides support that P. suffruticosa is a potential sole therapeutic or adjunct strategy for the treatment of triple-negative breast cancer patients.

\section{Acknowledgements}

The Authors would like to thank Bergen County Academies for providing necessary funds for executing this study and would like to thank Ms. Allison Gennaro for critical review of this manuscript.

\section{References}

1 Ginsburg, O and Love R: Breast cancer: a neglected disease for the majority of affected women worldwide. Breast J 17(3): 289295, 2011.

2 Brenton JD, Carey LA, Ahmed AA, Molecular classification and molecular forecasting of breast cancer: ready for clinical application? J Clin Oncol 29: 7350-7360, 2005.

3 Cui Y, Shu XO and Gao Y: Use of complementary and alternative medicine by Chinese women with breast cancer. Breast Cancer Res Treat 85(3): 263-370, 2004.

4 Ma H, Sullivan-Halley J, Smith AW, Neuhouser ML, Alfano CM, Meeske K, George SM, McTiernan A, McKean-Cowdin R, Baumgartner $\mathrm{KB}$, Ballard-Barbash $\mathrm{R}$ and Bernstein L: Estrogenic botanical supplements, health-related quality of life, fatigue, and hormone-related symptoms in breast cancer survivors: A heal study report. BMC Complement Altern Med 11: 109, 2011. 
5 Tian JH, Liu LS, Shi ZM, Zhou ZY and Wang L: A randomized controlled pilot trial of "feiji recipe" on quality of life of nonsmall cell lung cancer patients. Am J Chin Med 38(1): 15-25, 2010 .

6 Richardson MA, Sanders T, Palmer JL, Greisinger A and Singletary SE: Complementary/alternative medicine use in a comprehensive cancer center and the implications for oncology. J Clin Oncol 18(13): 2505-2514, 2000.

$7 \mathrm{Xu} \mathrm{Z}$, Chen X, Zhong Z, Chen L and Wang Y: Ganoderma lucidum polysaccharides: Immunomodulation and potential antitumor activities. Am J Chin Med 39(1): 15-27, 2011.

8 Choi HS, Seo HS, Kim JH, Um JY, Shin YC and Ko SG: Ethanol extract of paeonia suffruticosa andrews (pse) induced ags human gastric cancer cell apoptosis via fas-dependent apoptosis and mdm2-p53 pathways. J Biomed Sci 19(82), 2012.

9 Khan V, Najmi AK, Akhtar M, Aqil M, Mujeeb M and Pillai KK: A pharmacological appraisal of medicinal plants with antidiabetic potential. J Pharm Bioallied Sci 4(1): 27-42, 2012.

10 Wang SC, Tang SW, Lam SH, Wang CC, Liu YH, Lin HY, Lee SS and Lin JY: Aqueous extract of paeonia suffruticosa inhibits migration and metastasis of renal cell carcinoma cells via suppressing vegfr-3 pathway. Evid Based Complement Alternat Med 2012: 409823, 2012.

11 Miller JD, Clabaugh SE, Smith DR, Stevens RB and Wrenshall LE: Interleukin-2 is present in human blood vessels and released in biologically active form by heparanase. Immunol Cell Biol 90(2): 159-167, 2012.

12 Rose-John S: Il-6 trans-signaling via the soluble il-6 receptor: Importance for the pro-inflammatory activities of il-6. Int J Biol Sci 8(9): 1237-1247, 2012.

13 van Horssen R, Ten Hagen TL and Eggermont AM: Tnf-alpha in cancer treatment: Molecular insights, antitumor effects, and clinical utility. Oncologist 11(4): 397-408, 2006.

14 Jen EY, Poindexter NJ, Farnsworth ES and Grimm EA: Il-2 regulates the expression of the tumor suppressor il-24 in melanoma cells. Melanoma Res 22(1): 19-29, 2012.

15 Gopalan B, Litvak A, Sharma S, Mhashilkar AM, Chada S and Ramesh R: Activation of the fas-fasl signaling pathway by mda7/il-24 kills human ovarian cancer cells. Cancer Res 65(8): 3017-3024, 2005
16 Yacoub A, Liu R, Park MA, Hamed HA, Dash R, Schramm DN, Sarkar D, Dimitriev IP, Bell JK, Grant S, Farrell NP, Curiel DT, Fisher PB and Dent P: Cisplatin enhances protein kinase r-like endoplasmic reticulum kinase- and cd95-dependent melanoma differentiation-associated gene-7/interleukin-24-induced killing in ovarian carcinoma cells. Mol Pharmacol 77(2): 298-310, 2010.

17 Yaoxian W, Hui Y, Yunyan Z, Yanqin L, Xin G and Xiaoke W: Emodin induces apoptosis of human cervical cancer hela cells via intrinsic mitochondrial and extrinsic death receptor pathway. Cancer Cell Int 13(1): 71, 2013.

18 Esposito E and Cuzzocrea S: TNF-alpha as a therapeutic target in inflammatory diseases, ischemia-reperfusion injury and trauma. Curr Med Chem 16(24): 3152-3167, 2009.

19 Srivastava AN, Gupta A, Srivastava S, Natu SM, Mittal B, Negi MP and Prasad R: Cisplatin combination chemotherapy induces oxidative stress in advance non-small cell lung cancer patients. Asian Pac J Cancer Prev 11(2): 465-471, 2010.

20 Overwijk WW, Theoret MR and Restifo NP: The future of interleukin-2: Enhancing therapeutic anticancer vaccines. Cancer J Sci Am 6(Suppl 1): S76-80, 2000.

21 Radin D, Lippa A, Patel P and Leonardi D: Lifeguard inhibition of fas-mediated apoptosis: A possible mechanism for explaining the cisplatin resistance of triple-negative breast cancer cells. Biomed Pharmacother 77: 161-166, 2016.

22. Xie Y, Lv H, Sheng W, Miao J, Xiang J and Yang J: Synergistic tumor suppression by adenovirus-mediated inhibitor of growth 4 and interleukin-24 gene cotransfer in hepatocarcinoma cells. Cancer Biother Radiopharm 26(6): 681-695, 2011.

23 Krammer PH, Arnold R and Lavrik IN: Life and death in peripheral t cells. Nat Rev Immunol 7(7): 532-542, 2007.

Received June 7, 2017

Revised June 24, 2017

Accepted June 26, 2017 